\title{
Improving the Accuracy and Quality of the Surface Layer of Products by Local Magnetic Pulse Processing
}

\author{
Vasily Gritsyuk ${ }^{1}$, Evgeny Smolentsev ${ }^{2, *}$, Dmitriy Krokhin ${ }^{2}$, and Aleksandr Pechagin ${ }^{1}$ \\ ${ }^{1}$ KB Khimavtomatiki, 20 Voroshilov str., Voronezh 394055, Russia \\ ${ }^{2}$ Voronezh State Technical University, 14 Moskovskiy prospekt, Voronezh, Russia, 394026
}

\begin{abstract}
This article discusses development of technological modes and the process of stabilizing the properties of surface layers of large-sized parts, ensuring accuracy and stability of the products' shape during their manufacture and operation, while using combined processing methods that replace costly heat treatment at mechanical engineering enterprises.
\end{abstract}

The accuracy and stability of geometric dimensions of products, especially large-sized ones, the quality of the surface layer of materials determine the main performance indicators of products: operability, resource, efficiency of subsequent technological operations, and maintainability of objects. The indicators that determine accuracy and stability of a product's shape and properties of the surface layer are residual stresses in workpieces, which affect geometric parameters of products (especially large-sized ones) during the manufacture of parts and during their operation in products, in particular equipment and fixtures. Residual stresses can cause discontinuity of the material, its destruction or long-term change in the geometry of products. Mechanical processing of a workpiece reduces the magnitude and unevenness of stresses. However, in a number of cases, it is economically impractical to remove the allowance from individual sections of parts (for example, frames, bodies, machine stands), therefore, after local surface treatment, the stress unevenness increases even more compared to the same indicators for workpieces, and the deformation of products leads to a loss of accuracy and defective parts. To reduce distortion of products, stabilizing operations are used (for example, normalization, annealing), but for such operations with large-sized products, large thermal units are required, which can be located de away from the customer. The enterprises that produce special equipment, as a rule, do not have capacious furnaces, and fulfillment of orders for the manufacture of large body parts in another place is associated with significant costs for transportation, packaging, loading, heat treatment, which causes delays in the release of products, the need to store parts of products in the warehouse before the assembly stage. This increases their cost and can make such production uncompetitive.

To shorten the technological cycle and more fully utilize its own production facilities, it turned out to be promising to use the magnetic-pulse method of influencing the surface layer of cast and welded workpieces, which makes it possible to reduce and level out residual

\footnotetext{
* Corresponding author: smolentsev.rabota@gmail.com
} 
stresses that cause distortion of parts. However, with individual orders, the size and shape of the blanks of each product have significant differences, and a long-term correction of magnetic-pulse modes is required, which is practically impossible for single products. Therefore, a theoretical design base, experimental substantiation of the parameters for determining operating modes of magnetic-pulse processing, places of installation of inductors, the minimum number of influences to equalize the stresses in the surface layer of materials and the development of a typical technological process for the manufacture of cast and welded precise basic parts of equipment are required.

The aim of the work was to develop technological modes and a process for stabilizing the properties of surface layers of large-sized parts, ensuring accuracy and stability of the shape of products during their manufacture and operation by using energy magnetic-pulse effects that accelerate and localize stabilization and adequately replace costly and long-term methods of heat treatment at mechanical engineering enterprises.

The analysis of literature sources and experience in the manufacture of large-sized equipment with individual requirements showed that the available information on the influence of various types of stabilizing treatment on the change in the desired direction of residual stresses mainly covers materials related to the production of serial equipment and large-sized parts for other purposes, which, as a rule, earlier was carried out at enterprises equipped with the required thermal equipment; and after using it the internal structures of materials were stabilized and the required accuracy of products was ensured. The surface layer formation mechanism in the process of pulsed processing has been scarcely studied, although the controlled stabilization of the characteristics of the surface layer can provide the specified accuracy of the geometric shapes of large-sized parts during the period of their manufacture and operation in products. At the same time, there is no information on the serial production of equipment for electromagnetic stabilization of products, which necessitates the development of requirements for such installations, taking into account the peculiarities of manufacturing using one's own resources.

In the course of the study, a mechanism for stabilizing the geometry and obtaining the required properties of the surface layer during local magnetic-pulse processing was revealed.

A physical model of the process was developed, which showed that pulsed magnetic influences are capable of leveling residual stresses even without perceptible vibrations of the part.

The proposed mechanism is formalized by a system of equations that make up the model and allow the development of technological modes of the process.

Thus, depth $(T)$ of the zone of action of a known energy pulse can be estimated from the empirical dependence

$$
T=k_{n}\left\{k_{1} \frac{H_{\varphi}}{H_{x}}-F[\operatorname{arcSin}(\operatorname{Sin} \varphi / 2) / k \cdot K]\right\} .
$$

where $k_{1}, k_{n}, k$ are empirical coefficients; $K$ is the modulus of the residual stress distribution function; $H_{x}$ is field strength in the area of the pulse; $H_{\varphi}$ is field strength at the angle of rotation of its vector $(\varphi) ; F$ is the ratio of forces of external influence from the impulse to the internal resistance of the object.

Quantity $T \geq h_{3}$, where $h_{3}$ is the minimum penetration depth of the field, calculated from the dependence

$$
h_{3} \geq \frac{\sqrt[4]{L C}}{\sqrt{0,5 \gamma_{3} \mu_{3}}},
$$

where $\gamma_{3}$ is the conductivity of the workpiece material; $\mu_{3}$ is the magnetic constant for workpiece material; $L$ is discharge circuit inductance; $C$ is the condenser capacity. 
Then the thickness of the part in the processing zone at the selected pulse energy must be at least $h_{3}$. If this condition is not met, the pulse energy should be changed.

$$
\tau=0,5 \pi \sqrt{L C}
$$

Numerical calculations using the model made it possible to establish the depth of the pulse action zone and confirm this experimentally, which is shown in Fig. 1.

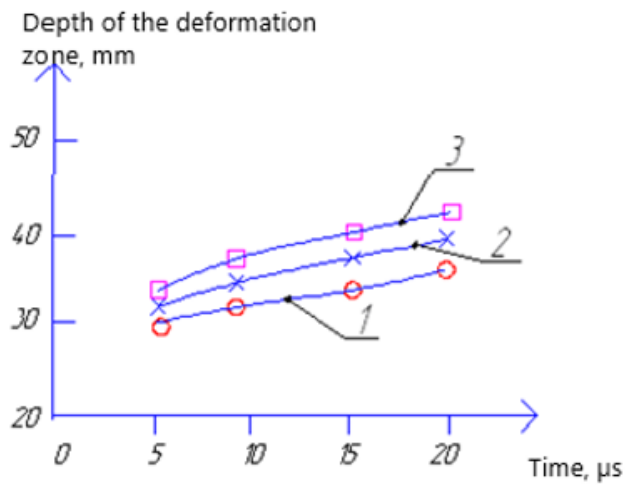

Fig. 1. Depth of the operating range of pulses with their duration: 1 - $5 \mu \mathrm{s} ; 2$ - $10 \mu \mathrm{s} ; 3-100 \mu \mathrm{s}$ (experimental points are plotted on the calculated dependences)

The analysis of Fig. 1 confirms validity of the proposed model and the mechanism of the impulse action. A fairly high convergence is observed here.

The experimental studies for thick-walled samples from various materials, shown in Fig. 2 , confirm the effectiveness of magnetic-pulse stabilization when using technological modes calculated from the dependencies of the process model.

The technological process contains several stages.

At the first stage, the source data are selected, which take into account:

1. Information about the workpiece: dimensions; geometry of areas requiring dimensional stabilization; material grade; method of obtaining a workpiece; geometrical dimensions of inductor installation sites, etc.

2. Technological requirements for the workpiece and parts: limiting, economically justified, allowances; mechanical stripping of areas to be stabilized; part dimensional tolerances; roughness and waviness of contact surfaces; absence of grooves, breaks, recesses on the surface of the workpiece that distort the field.

3. Technological capabilities of the available equipment: the number of simultaneously operating inductors; the highest energy indicators of generators; resource of emitters; availability of personnel for qualified maintenance of installations. 


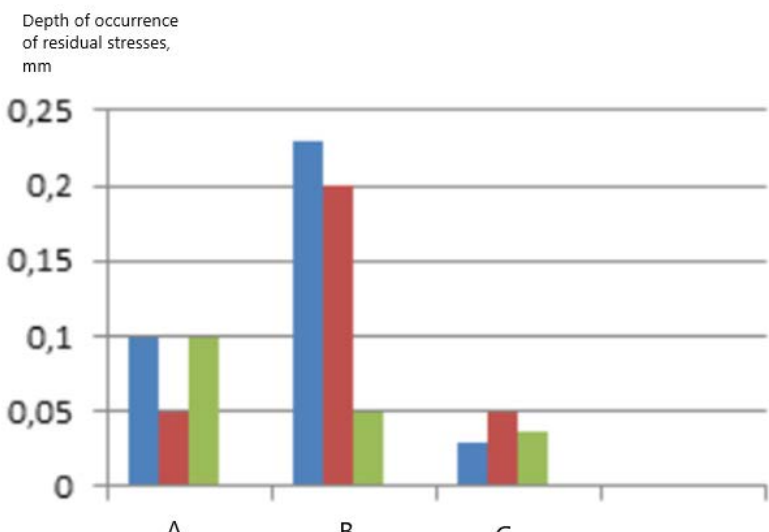

A

a)

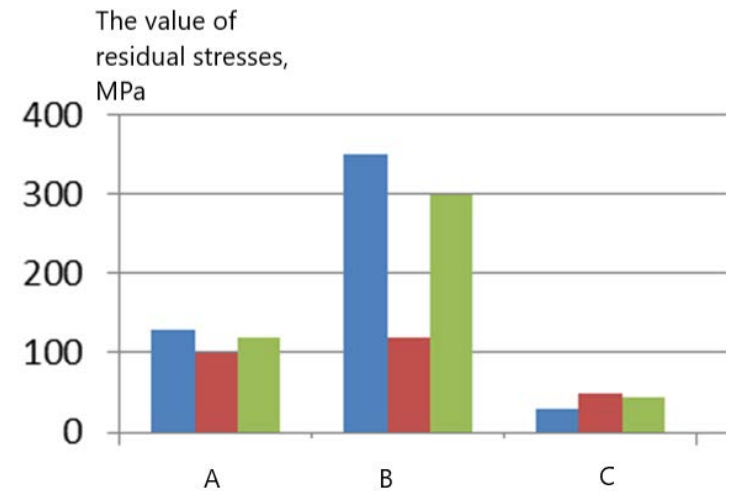

b)

Fig. 2. Change in the depth of occurrence (a) and the magnitude of residual stresses (b) of steel (1), cast iron (2), titanium (3) alloys: A - welding; B - casting; C - magnetic pulse processing

The first stage of the process includes:

- classification of sections of the surfaces of blanks according to a geometric feature (flat, box-shaped with constant and variable sections, etc.);

- $\quad$ selection or necessity of designing and manufacturing inductors and adapters. Experiments have shown that the working parts of inductors must be up to $250 \mathrm{~mm}$ in size;

- $\quad$ the need to move the workpiece or reinstall inductors (adapters);

- calculation of allowances for stripping parts and size of the removed material after magnetic-pulse processing;

- $\quad$ assignment of the amount of time for natural stabilization of internal stresses after magnetic-pulse processing before the start of finishing operations;

- calculation or experimental determination of residual stresses and changes in geometry under the action of operational loads. The limiting values of errors in shape and dimensions are regulated by technical documentation for the part.

At the next stage, the processing modes are calculated:

- placement of inductors (with or without adapters, with a contact medium).

- calculation of allowances $\left(Z_{0}\right)$ for the finishing stage of machining after magneticpulse stabilization

$$
Z_{0}=\delta_{0}+\left(R_{Z}+T\right)_{n}+\delta_{n}+\delta_{\partial}
$$


where $\delta_{0}$ is the permissible error after the final stage of processing a part; $\left(R_{z}+T\right)_{n}$ is the height of irregularities and changed layer after preliminary processing (roughing) of the workpiece; $\delta_{n}$ is the maximum permissible error after preprocessing; $\delta_{\partial}$ is the limiting change in the geometry of the part in the process of 'aging' (natural aging);

- calculation of the limiting pulse energy. It is carried out from the condition of obtaining stresses $\left(\sigma_{\max }\right)$ from an electromagnetic pulse that do not exceed the ultimate strength of the part material $\left(\sigma_{\hat{a}}\right)$

$$
\sigma_{\max }<\sigma_{\hat{a}}\left(\sigma_{\mathrm{m} \text { a }}=K_{3} \cdot \sigma_{\theta}\right),
$$

where $K_{3}$ is the safety factor taking into account random factors $\left(K_{3}=0,8-0,85\right)$.

Then the density of the surface forces of the electromagnetic pulse $\left(P_{U}\right)$ is going to be:

- for parts such as beams and box structures

$$
P_{U}=K_{3} \sigma_{\theta} \cdot h l \cdot k_{\mathrm{n}},
$$

- for flat parts such as slabs

$$
P_{U}=K_{3} \sigma_{6} \cdot h \cdot k_{p}^{x},
$$

where $h$ is thickness or height of the section of a rectangular section part. For other shapes, through the equality of the moments of inertia of the section under consideration, the equivalent thickness or height of the section used in the calculations is found; $l$ is the section width of the part or the wide part of the box-shaped section; $k_{\mathrm{n}}$ is the coefficient taking into account the size of the processing area and parts, placement of inductors for beams and box-shaped parts; $k_{p}$ is the coefficient for flat parts; $x$ is the power coefficient taking into account placement of inductors and the distance between them.

The density of the surface forces of an electromagnetic pulse depends on its energy $\left(A_{U}\right)$

$$
P_{U}=K_{U} \cdot A_{U}
$$

where $K_{U}$ is the coefficient that takes into account transition of the shock wave energy into a force pulse, energy losses, the size and uniformity of the gaps between the part and the inductor (adapter).

For generators with a pulsed discharge, the pulse energy is

$$
A_{U}=\frac{C U_{n p}^{₹}}{2},
$$

where $U_{n p}$ - breakdown voltage, selectable within $U_{n p}=0,7 U\left(U=(1 \div 20) 10^{3} B\right)$;

$C$ - capacitance of capacitors $(\mu \mathrm{F})$, technological parameter;

- for beams and box parts

- for flat parts

$$
C=\frac{2 K_{3} k_{\mathrm{n}} \sigma_{b} h l}{K_{u} U_{n p}^{2}},
$$

$$
C=\frac{2 K_{3} \kappa_{p}^{x} \sigma_{b} h}{K_{u} U_{n p}^{2}} .
$$

Next, pulse repetition frequency $(v)$ is found 


$$
v=\frac{1}{2,3 R \mathbb{C g} \frac{U-U_{k}}{U-U_{n p}},}
$$

where $R$ is the loop resistance determined through voltage $(U)$ and current $\left(J_{u}\right)$ realized in the pulse

$$
R=\frac{U}{J_{u}},
$$

$U_{\kappa}$ is the voltage at the end of the pulse (may not be taken into account in the calculations).

Then approximately

$$
v=\frac{0,837}{R C} .
$$

Hence, the pulse duration $\left(\tau_{u}\right)$

$$
\tau_{U}=\frac{\mathrm{q} \cdot R C}{0,83 \tau}
$$

where $\mathrm{q}$ is relative pulse duration.

The number of pulses $(n)$ during magnetic pulse stabilization is at least 10-15. Then machine time $(t)$ of the operation will be

$$
t=\left(\tau_{u}+\tau_{0}\right) \cdot n+\tau_{n} \cdot m
$$

where $\tau_{0}$ is the pause time between pulses; $\tau_{0}$ is the inductor reset time; $m$ is the number of redeployment of inductors when processing a large-sized part.

At the end of the operation, the amount of deformation of the part is monitored. $(\Delta \delta)$.

$$
\Delta \delta \leq\left[\delta_{0}\right],
$$

where $\left(\left[\delta_{0}\right]\right)$ is the permissible value of the change in the geometric dimensions of the parts after stabilization.

The fifth chapter provides a rationale for the capabilities and scope of magnetic pulse stabilization.

The dynamics of reduction of residual stresses to the required level in cast iron (Fig. 3, a) and steel welded products (Fig. 3, b) was investigated.

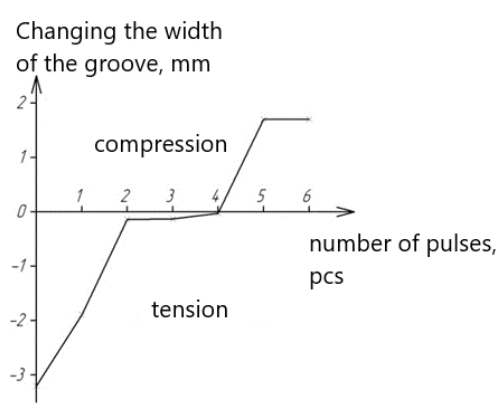

a)

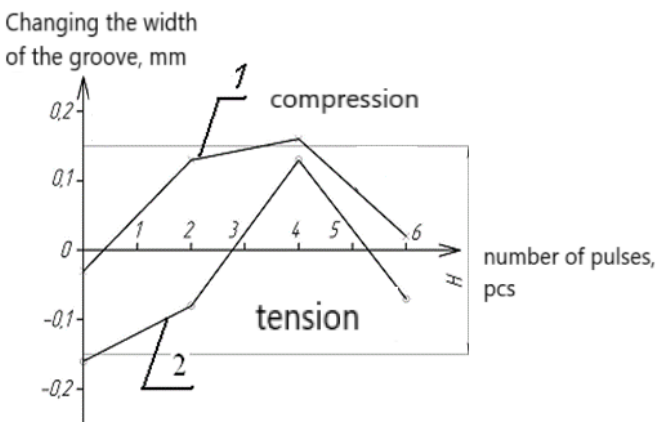

b)

Fig. 3. Change in the groove width, characterizing the value of residual stresses in the surface layers of alloys, from the number of pulses 
In Fig. 3, it can be seen that, depending on the energy of a single pulse, the number of pulses can be reduced to 5-6, and taking into account the stabilization natural redistribution of stresses in the surface layer, it can be recommended when calculating the modes in the number from 10 to 18 . This makes it possible to refine the choice of the process parameters given in the third chapter. The pauses between pulses are necessary to limit the temperature of the treatment area. The experiments have shown that at pulse energies below $5 \mathrm{~kJ}$ this time interval is 2-3 seconds, and at more powerful pulses - up to 7 seconds.

Comparison of technical and economic indicators for the manufacture of typical largesized parts at enterprises with different structural organization showed that the magneticpulse stabilization of parts gives a significant economic benefit, especially for small and medium-sized businesses.

\section{References}

1. Smolentsev V.P., Smolentsev E.V., The state and development prospects of combined processing methods, Bulletin of the Rybinsk State Aviation Technological Academy named after P.A. Solovyev, 2017. No. 2 (41). pp. 5-9.

2. A.A. Klimenchenkov, E.V. Smolentsev, Formation of micro and nanoprofiles of mating surfaces during combined processing, Nanoinzhenerija Publ., 2013, No. 7, pp. 8-15

3. Ryazantsev A., Yukhnevich S, Use of combined methods of treatment to obtain artificial roughness on the parts' surfaces, MATEC Web of Conferences: International Conference on Modern Trends in Manufacturing Technologies and Equipment (ICMTMTE 2018), 224, 01058 (2018) DOI: 10.1051/matecconf/201822401058

4. Shirokozhukhova A., Ryazantsev A., Gritsyuk V., Science-based technologies creation based on combined processing methods for fabrication aerospace filters, Materials Today: Proceedings Volume 19, Part 5, 2019, Pages 2065-2067 https://doi.org/10.1016/j.matpr.2019.07.075

5. Ablyaz, T.R.; Bains,P.S.; Sidhu, S.S.; Muratov, K.R.; Shlykov, E.S. Impact of Magnetic Field Environment on the EDM Performance of Al-SiC Metal Matrix Composite. Micromachines 2021, 12, 469. https://doi.org/10.3390/ mi12050469

6. Surface Characterization and Tribological Performance Analysis of Electric Discharge Machined Duplex Stainless Steel / T. R. Ablyaz, E. S. Shlykov, K. R. Muratov, A. Mahajan, G. Singh, S. Devgan, S. S. Sidhu // Micromachines [Electronic resource]. 2020. - Vol. 11, Iss. 10. - Art. 926. - 14 p. 